\title{
Relative Spectral Norm on Algebraic Numbers
}

\author{
Angel Popescu (*) - Sobia Sultana (**)
}

ABstRact - Let $K$ be a subfield of $\bar{Q}$, a fixed algebraic closure of Q, the field of rational numbers. Let $G_{K}=\operatorname{Gal}(\overline{\mathrm{Q}} / K)$ be the absolute Galois group of $K$. For any $x \in \overline{\mathrm{Q}}$, we consider the $K$-spectral norm: $\|x\|_{K}=\max \left\{|\sigma(x)|: \sigma \in G_{K}\right\}$. Let $\bar{e}$ be the conjugation automorphism of $\bar{Q}$ and let $\mathcal{C}\left(G_{K}\right)$ be the Banach algebra of all continuous mappings defined on the compact group $G_{K}$ with values in C. Let $\mathcal{C}_{\text {sym }}\left(G_{K}\right)$ be the Banach algebra over $\widetilde{K}$ of all symmetric functions of $\mathcal{C}\left(G_{K}\right)$. Here $\widetilde{K}=\mathbb{R}$ or $\mathbb{C}$ is the completion of $K$ relative to the usual absolute value |.|. A function $f$ is said to be symmetric if $f(\bar{e} \sigma)=\bar{e}(f(\sigma))$ for any $\sigma \in G_{K}$ (when $\bar{e} \in G_{K}$ ). Let $\widetilde{Q}_{K}$ be the completion of $\bar{Q}$ with respect to $\|\cdot\|_{K}$. In this paper we prove that $\widetilde{Q}_{K} \cong \mathcal{C}_{\text {sym }}\left(G_{K}\right)$ if $\bar{e} \in G_{K}$ and $\widetilde{Q}_{K} \cong \mathcal{C}\left(G_{K}\right)$ if $\bar{e} \notin G_{K}$. These last isomorphisms are $\widetilde{K}$-isomorphisms of Banach algebras. We give some other properties of the closed subalgebras of $\mathcal{C}\left(G_{K}\right)$ in connection with some subfields of algebraic numbers.

1. The notion of a spectral norm on the algebraic number field was introduced by A. Popescu, N. Popescu and A. Zaharescu in [13]. This is a norm $\|$.$\| on \bar{Q}$, a fixed algebraic closure of $\mathrm{Q}$, the field of rational numbers, defined by $x \longrightarrow\|x\|=\max \{|\sigma(x)|: \sigma \in \operatorname{Gal}(\overline{\mathrm{Q}} / \mathrm{Q})\}$. The completion $\widetilde{\mathrm{Q}}$ of $\overline{\mathrm{Q}}$ relative to this norm is a ring with zero divisors but with some interesting arithmetical and topological properties (see [8], [10], [11] and [13] - [17]). The main property of $\widetilde{Q}$ is that the absolute Galois group $G=\operatorname{Gal}(\overline{\mathrm{Q}} / \mathrm{Q})$ of $Q$ acts continuously on it, which is not the case of $\mathrm{C}$ (only the identity $e$ and the conjugation automorphism $\bar{e}$ of $G$ act continuously on C). So that, if we

(*) Indirizzo dell'A.: Technical University of Civil Engineering Bucharest, Department of Mathematics, B-ul Lacul Tei 124, 72302 Bucharest 38, Romania.

E-mail: angel.popescu@gmail.com

(**) Indirizzo dell'A.: Abdus Salam School of Mathematical Sciences, 68-B, New Muslim Town, Lahore, Pakistan.

E-mail: sobia.sultana@gmail.com

1991 Mathematics Subject Classification. Primary 11R99, 12J10; Secondary 46J10, 11R32. 
want to get information on $G$ by its action on different topological spaces, a good candidate is $\widetilde{Q}$. Such type of researches were also done for the local absolute Galois group $G_{p}=\operatorname{Gal}\left(\overline{\mathrm{Q}}_{p} / \mathrm{Q}_{p}\right)$, where $\overline{\mathrm{Q}}_{p}$ is a fixed algebraic closure of the $p$-adic number field $Q_{p}$ (see [4], [9], and [12]). Some ideas of these researches come directly or indirectly from [2], [3].

In a letter to the first author [19], Prof. A. Zaharescu had the idea to generalize the usual spectral norm $\|$.$\| to a K$-spectral norm on $\overline{\mathrm{Q}}$ :

$$
x \longrightarrow\|x\|_{K}=\max \left\{|\sigma(x)|: \sigma \in G_{K}=\operatorname{Gal}(\overline{\mathrm{Q}} / K)\right\},
$$

where $K$ is an arbitrary subfield of $\bar{Q}$.

In this paper we study the completion $\widetilde{\mathrm{Q}}_{K}$ of $\overline{\mathrm{Q}}$ with respect to the $K$ spectral norm defined above. Since the techniques of [13] - [17] are sometimes not useful in this new frame, we introduce some new ones. Let $\mathcal{C}\left(G_{K}\right)$ be the $\mathrm{C}$-Banach algebra of all continuous mappings defined on the compact group $G_{K}=\operatorname{Gal}(\overline{\mathrm{Q}} / K)$ (relative to its Krull topology) with values in $\mathbb{C}$ (with its usual absolute value topology). An element $f \in \mathcal{C}\left(G_{K}\right)$ is said to be symmetric (only in the case when $\bar{e} \in G_{K}$ ) if $f(\bar{e} \sigma)=\bar{e}(f(\sigma)$ ) for any $\sigma \in G_{K}$. If $\bar{e} \notin G_{K}$ we cannot define such a notion. Let $\mathcal{C}_{\text {sym }}\left(G_{K}\right)$ be the $K$ subalgebra of $\mathcal{C}\left(G_{K}\right)$ which consists of all symmetric mappings of $\mathcal{C}\left(G_{K}\right)$. Let $\widetilde{L}_{K}$ be the topological closure in $\widetilde{\mathrm{Q}}_{K}$ of a subfield $L$ of $\overline{\mathrm{Q}}, L \supset K$, with respect to $\|.\|_{K}$. In Theorem 1 and Remark 2 we prove that if $K \subseteq \mathbb{R} \cap \bar{Q}$, then $\widetilde{\mathrm{Q}}_{K}$ is isomorphic and isometric with $\mathcal{C}_{\text {sym }}\left(G_{K}\right)$ as $\widetilde{K}$-algebras. If $K \nsubseteq \mathbb{R} \cap \overline{\mathrm{Q}}$ ( i.e. $\bar{e} \notin G_{K}$ ), then $\widetilde{\mathrm{Q}}_{K}$ is isometric and isomorphic as C-algebras with $\mathcal{C}\left(G_{K}\right)$ (Theorem 2). In Theorem 3 we prove that always $\widetilde{Q}_{K}[\widetilde{i}] \cong \mathcal{C}\left(G_{K}\right)$, where $\tilde{i}$ is the constant function $f(\sigma)=i=\sqrt{-1}$ for any $\sigma$ in $G_{K}$. So $\bar{\varphi}\left(\widetilde{Q}_{K}\right)$ has the codimension 1 or 2 in $\mathcal{C}\left(G_{K}\right)$. Here $\varphi: \bar{Q} \longrightarrow \mathcal{C}\left(G_{K}\right)$, is the following map: $\varphi(\alpha)=\varphi_{\alpha}$, where $\varphi_{\alpha}(\sigma)=\sigma(\alpha)$ and $\bar{\varphi}$ is the unique extension by continuity of $\varphi$ to $\widetilde{\mathrm{Q}}_{K}$. In Theorems 4,5 and 6 we obtain analogous results as in Theorems 1,2 and 3 for a subextension $L / K$ of $\overline{\mathrm{Q}} / K$. Theorem 7 is the direct generalization of Theorem 2.2 of [13], namely $\widetilde{L}_{K}=\widetilde{K[x]}$ for any $K \subset L \subset \overline{\mathrm{Q}}$ and for an $x \in \widetilde{L}_{K}$ (a generalization of the primitive element theorem from the classical field theory). In Lemma 2 and Corollary 2 we give a general Archimedean frame for the classical idea of Krasner (Krasner's Lemma) [1] or [7]. We apply this new ideas to prove that if $f, g$ are locally constant and nonconstant mapping from $\mathcal{C}\left(G_{K}\right)$ such that $g$ is "sufficiently close" to $f$, then $\mathbb{C}[f] \subset \mathbb{C}[g]$ (see Theorem 8). Then we apply this last result in order to get some information on the closed $\widetilde{K}$ subalgebras of $\mathcal{C}\left(G_{K}\right)$ (see Corollary 3, Proposition 2 and Proposition 3).

2. Let $\mathrm{Q}$ be the rational number field and let $\mathrm{C}$ be a fixed complex number field, $\mathrm{Q} \subset \mathrm{C}$. Let $\overline{\mathrm{Q}}$ be the algebraic closure of $\mathrm{Q}$ in $\mathrm{C}$ and let $K$, 
$\mathrm{Q} \subset K \subset \overline{\mathrm{Q}}$ be a fixed subfield of $\overline{\mathrm{Q}}$. If $[K: \mathrm{Q}]<\infty$, usually $K$ is called an algebraic number field. In this paper we call any subfield of $\overline{\mathrm{Q}}$ an algebraic number field. Let $G_{K}=\operatorname{Gal}(\overline{\mathrm{Q}} / K)$ be the absolute Galois group of $K$. We denote by $G=\operatorname{Gal}(\overline{\mathrm{Q}} / \mathrm{Q})$ the absolute Galois group of $\mathrm{Q}$. $G$ is a compact totally disconnected group relative to the Krull topology on it [1]. The Krull topology on $G_{K} \subset G$ is exactly the topology of $G$ induced to $G_{K}$. If $G_{K}$ is finite, the Krull topology becomes the discrete topology.

Let us fix a tower of subfields:

$$
K=K_{0} \subset K_{1} \subset K_{2} \subset \ldots \subset K_{n} \subset \ldots \subset \bar{Q},
$$

such that all extensions $K_{n} / K$ are normal and finite and $\cup_{n=0}^{\infty} K_{n}=\overline{\mathrm{Q}}$. Then the Galois groups $H_{n}=\operatorname{Gal}\left(\overline{\mathrm{Q}} / K_{n}\right), n=0,1,2, \ldots$ are closed and open and form a fundamental system of neighborhoods of the identity automorphism $e$ of $G_{K}$. Let denote by $|$.$| the usual absolute value on C. For any$ $\sigma \in G_{K}, x \longrightarrow|\sigma(x)|=|x|_{\sigma}$ is an Archimedean absolute value on $\bar{Q}$. Let $\bar{e}$ be the conjugation automorphism of $\overline{\mathrm{Q}}$. If $\bar{e} \in G_{K}$ then $|\cdot|_{\bar{e} \sigma}=|\cdot|_{\sigma}$. So any two automorphisms $\sigma, \mu \in G_{K}$ give rise to one and the same topology on $\bar{Q}\left(|\cdot|_{\sigma}\right.$ and $|\cdot|_{\mu}$ are dependent) if and only if $\sigma=\bar{e} \mu$. For any $x \in \overline{\mathrm{Q}}$ one considers:

$$
\|x\|_{K}=\max \left\{|\sigma(x)|: \sigma \in G_{K}\right\} .
$$

It is not difficult to prove the following result.

LEMma 1. The $\operatorname{map} x \longrightarrow\|x\|_{K}$ is a norm on $\overline{\mathrm{Q}}$ and $\left(\overline{\mathrm{Q}},\|.\|_{K}\right)$ is a $K$ normed algebra, i.e.:

i) $\|x\|_{K}=0$ if and only if $x=0$, for any $x \in \bar{Q}$,

ii) $\|x y\|_{K} \leq\|x\|_{K}\|y\|_{K}$, for any $x, y \in \overline{\mathrm{Q}}$,

iii) $\|x+y\|_{K} \leq\|x\|_{K}+\|y\|_{K}$, for any $x, y \in \bar{Q}$,

iv) $\|\alpha x\|_{K}=|\alpha|\|x\|_{K}$, for $\alpha \in K, x \in \bar{Q}$, and

v) $\|1\|_{K}=1$.

We call this norm the $K$-spectral norm on $\bar{Q}$. It extends to $\overline{\mathrm{Q}}$ the usual absolute value of $K$. In general, in ii), one has no equality. For instance, if $K=\mathbb{Q}$ and $x=1+\sqrt{2}, y=1-\sqrt{2} \in \bar{Q}$, then $\|x y\|_{K}=1 \neq(1+\sqrt{2})^{2}=$ $=\|1+\sqrt{2}\|_{K}\|1-\sqrt{2}\|_{K}$. Taking count of the Artin-Schreir theory [6] we see that the $K$-spectral norm $x \longrightarrow\|\sigma(x)\|_{K}$ is an absolute value on $\bar{Q}$ equal to the usual absolute value if and only if $K$ is a "real closed" subfield in $\bar{Q}$, i.e. if $K(i)=\overline{\mathrm{Q}}$. In this last case $G_{K} \cong Z_{2}$ and this case is the only case when $G_{K}$ is finite. Let $\widetilde{Q}_{K}$ be the completion of $\bar{Q}$ relative to the $K$-spectral norm. The topological closure $\widetilde{K}$ of $K$ in $\widetilde{Q}_{K}$ is $\mathbb{R}$ or $C$ whenever $K \subset \mathbb{R} \cap \overline{\mathrm{Q}}$ or not. Hence $K$ is dense in $\mathbb{C}$ (relative to $|$.$| ) if and only if K \nsubseteq \mathbb{R} \cap \overline{\mathrm{Q}}$. In 
general $\widetilde{Q}_{K}$ has zero divisors. It is a field if and only if $K$ is a "real closed" subfield in $\overline{\mathrm{Q}}$. In all the other cases $\widetilde{\mathrm{Q}}_{K}$ has zero divisors. But always $\widetilde{\mathrm{Q}}_{K}$ is a $\widetilde{K}$-Banach algebra.

Let us now consider the C-Banach algebra

$$
\mathcal{C}\left(G_{K}\right)=\left\{f: G_{K} \longrightarrow \mathbb{C} \mid f \text { continuous }\right\},
$$

with the usual sup-norm: $\|f\|=\sup \left\{|f(\sigma)|: \sigma \in G_{K}\right\}$. Since $G_{K}$ is a compact group, this last quantity is always finite. For any $\alpha \in \bar{Q}$ one defines :

$$
\varphi_{\alpha}: G_{K} \longrightarrow \mathrm{C}, \varphi_{\alpha}(\sigma)=\sigma(\alpha) .
$$

The map $\varphi_{\alpha}$ has only a finite number of values namely, the image of $\varphi_{\alpha}$ is the orbit of $\alpha$ (relative to $G_{K}$ ), i.e. the set of all conjugates of $\alpha$ in $\bar{Q}$. If $\alpha \in K, \varphi_{\alpha}(\sigma)=\alpha$ for any $\sigma \in G_{K}$. So we can embed $\widetilde{K}$ into $\mathcal{C}\left(G_{K}\right)$ by associating to $\alpha \in K$, the constant map $\varphi_{\alpha}$ in $\mathcal{C}\left(G_{K}\right)$.

Proposition 1. The map $\varphi: \overline{\mathrm{Q}} \longrightarrow \mathcal{C}\left(G_{K}\right)$ defined by $\varphi(\alpha)=\varphi_{\alpha}, \varphi_{\alpha}(\sigma)=$ $=\sigma(\alpha)$ is an isometry and a $K$-algebra morphism. So it is a $K$-embedding of $\left(\overline{\mathrm{Q}},\|\cdot\|_{K}\right)$ into $\left(\mathcal{C}\left(G_{K}\right),\|\cdot\|\right)$.

PRoof. It is clear that $\varphi_{\alpha}+\varphi_{\beta}=\varphi_{\alpha+\beta}, \varphi_{\alpha} \varphi_{\beta}=\varphi_{\alpha \beta}$ and $\lambda \varphi_{\alpha}=\varphi_{\lambda \alpha}$ for any $\alpha, \beta \in \overline{\mathrm{Q}}$ and $\lambda \in K$. Now $\|\alpha\|_{K}=\max \left\{|\sigma(\alpha)|: \sigma \in G_{K}\right\}=$ $=\sup \left\{\left|\varphi_{\alpha}(\sigma)\right|: \sigma \in G_{K}\right\}=\left\|\varphi_{\alpha}\right\|$ and the proof is complete.

For some later applications we fix $\alpha \in \overline{\mathrm{Q}}$ and a $\sigma_{0} \in G_{K}$. We are going to describe $\varphi_{\alpha}^{-1}\left(\sigma_{0}(\alpha)\right)$. If $\alpha \in K, \varphi_{\alpha}^{-1}\left(\sigma_{0}(\alpha)\right)=G_{K}$. If $\alpha \notin K$, let us take $L=K(\alpha) \subset \bar{Q}$ and let us consider $H_{L}$ the corresponding subgroup of $L$ : $H_{L}=\left\{\mu \in G_{K}: \mu(\alpha)=\alpha\right\}$. Then $\varphi_{\alpha}^{-1}\left(\sigma_{0}(\alpha)\right)=\sigma_{0} H_{L}$, the left coset of $\sigma_{0}$ in $G_{K} / H_{L}$. Since $H_{L}$ has the index $m=\operatorname{deg}_{K} \alpha, H_{L}$ is closed and open in $G_{K}$. Hence $\sigma_{0} H_{L}$ is also closed and open. It follows that $\varphi_{\alpha}$ is a continuous map which has the same value on each $\sigma_{i} H_{L}$, where $G_{K}=e H_{L} \cup \sigma_{2} H_{L} \cup \ldots \cup \sigma_{m} H_{L}$ is the partition of $G_{K}$, corresponding to the left classes of $G_{K} / H_{L}$.

COROLLARY 1. There is one and only one isometry and $\widetilde{K}$-algebra morphism $\bar{\varphi}$ which extend by continuity $\varphi$ to $\widetilde{\mathbb{Q}}_{K}: \bar{\varphi}(f)=\lim _{n \rightarrow \infty} \varphi_{\alpha_{n}}$, where $f \in \widetilde{Q}_{K}, f=\lim _{n \rightarrow \infty} \alpha_{n}$ relative to $\|\cdot\|_{K}$ and $\alpha_{n} \in \overline{\mathrm{Q}}, n=1,2, \ldots$

REMARK 1. Since $\|\sigma(\alpha)\|_{K}=\|\alpha\|_{K}$ for any $\sigma \in G_{K}$ and $\alpha \in \bar{Q}$, any $\sigma \in G_{K}$ is a continuous automorphism of $\overline{\mathrm{Q}}$ relative to $\|\cdot\|_{K}$. This is the main reason to consider the $K$-spectral norm on $\overline{\mathrm{Q}}$ instead of usual absolute 
value |.|. If $\left\{\alpha_{n}\right\}_{n}$ is a Cauchy sequence in $\bar{Q}$ relative to $\|.\|_{K}$, then $\left\{\sigma\left(\alpha_{n}\right)\right\}_{n}$ is also a Cauchy sequence in $\bar{Q}$ relative to the same norm. So the element $\sigma\left(A=\lim _{n \rightarrow \infty} \alpha_{n}\right)=\lim _{n \rightarrow \infty} \sigma\left(\alpha_{n}\right)$ does exist in $\widetilde{\mathrm{Q}}_{K}$. We denote it by $\sigma(A)$. If $f_{n} \stackrel{\|\cdot\|}{\rightarrow} f$ in $\mathcal{C}\left(G_{K}\right)$ then $\|f\|=\lim _{n \rightarrow \infty}\left\|f_{n}\right\|$, so $\|\sigma(A)\|_{K}=\|A\|_{K}$ for any $A \in \widetilde{\mathbb{Q}}_{K}$. Moreover $\|A\|_{K}=\lim _{n \rightarrow \infty}\left\|\alpha_{n}\right\|_{K}$.

Sometimes we identify $\widetilde{\mathbb{Q}}_{K}$ with its image $\bar{\varphi}\left(\widetilde{Q}_{K}\right)$ in $\mathcal{C}\left(G_{K}\right)$.

Definition 1. (see also [13]). Assume that $\bar{e} \in G_{K}$. A map $f: G_{K} \longrightarrow \mathbb{C}$ is said to be symmetric if $f(\bar{e} \sigma)=\overline{f(\sigma)}$, where $\overline{f(\sigma)}$ is the usual conjugate of $f(\sigma)$ in $\mathrm{C}$ and $\sigma$ is any element of $G_{K}$.

The $\widetilde{K}$-subalgebra of all symmetric maps of $\mathcal{C}\left(G_{K}\right)$ is denoted by $\mathcal{C}_{\text {sym }}\left(G_{K}\right)$. It is closed in $\mathcal{C}\left(G_{K}\right)$, so it is a $\widetilde{K}$-Banach subalgebra of $\mathcal{C}\left(G_{K}\right)$.

TheOREM 1. Let $K$ be a proper subfield of $\mathbb{R} \cap \overline{\mathrm{Q}}$. Then $\widetilde{\mathrm{Q}}_{K}$ is $\widetilde{K}$-isomorphic and isometric with $\mathcal{C}_{\text {sym }}\left(G_{K}\right)$.

Proof. Since $\bar{e} \in G_{K}$ in this case $\left(\bar{e} \in G_{K}\right.$ if and only if $\left.K \subset \mathbb{R} \cap \bar{Q}\right)$, we can use the same technique as in [13]. However we prefer here a direct proof, which could be useful in other future researches. First of all we must remark that $\varphi_{\alpha}$, for any $\alpha \in \overline{\mathrm{Q}}$, is a symmetric continuous map. Since any $x \in \widetilde{\mathrm{Q}}_{K}$ is a limit of $x_{n} \in \overline{\mathrm{Q}}$ with respect to \|\|$_{K}$, one has that $\varphi_{x_{n}} \rightarrow \varphi_{x}$ in $\mathcal{C}\left(G_{K}\right)$, where $\varphi_{x}(\sigma)=\sigma(x)$ (see Remark 1 ). One can use the fact that the conjugation automorphism $\bar{e}$ is continuous relative to $|$.$| on \mathrm{C}$. So $\left(\lim _{n \rightarrow \infty} \varphi_{x_{n}}\right)(\bar{e} \sigma)=\bar{e}\left(\lim _{n \rightarrow \infty} \varphi_{x_{n}}(\sigma)\right)=\varlimsup_{n \rightarrow \infty} \varphi_{x_{n}}(\sigma)$, i.e. $\varphi_{x} \in \mathcal{C}_{\text {sym }}\left(G_{K}\right)$. We prove now that any element $f$ of $\mathcal{C}_{\text {sym }}\left(G_{K}\right)$ can be approximated by "algebraic numbers" $\varphi_{x}$, where $x \in \overline{\mathrm{Q}}$. Let $f$ be in $\mathcal{C}_{\text {sym }}\left(G_{K}\right)$ and let $\varepsilon>0$ be a small positive real number. Let $H$ be a finite index subgroup of $G_{K}, \bar{e} \notin H$ and such that if $G_{K}=\sigma_{1} H \cup \ldots \cup \sigma_{m} H, \sigma_{1}=e$, is the corresponding partition of $G_{K}$ into left cosets, one has $|f(\sigma)-f(\mu)|<\frac{\varepsilon}{3}$ whenever $\sigma, \mu \in \sigma_{i} H$ for an $i \in\{1,2, \ldots, n\}$. Let us write $\lambda_{i}=f\left(\sigma_{i}\right)$, for $i=1,2, \ldots, n$. Since $\bar{e} \in G_{K}$ we can divide $\sigma_{1}, \ldots, \sigma_{m}$ into two classes relative to the subgroup $\{e, \bar{e}\}$ :

$$
\left\{\sigma_{1}, \ldots, \sigma_{m}\right\}=\left\{\sigma_{1}, \ldots, \sigma_{t}, \sigma_{t+1}=\bar{e} \sigma_{1}, \ldots, \sigma_{2 t}=\bar{e} \sigma_{t}\right\}, m=2 t
$$

Since $f\left(\bar{e} \sigma_{j}\right)=\overline{f\left(\sigma_{j}\right)}, j=1,2, \ldots, t$, one has that $\lambda_{t+h}=\bar{\lambda}_{h}$ for any $h=1,2, \ldots, t$. We focus only on the points $\lambda_{1}, \lambda_{2}, \ldots, \lambda_{t}$ in the complex plane $\mathrm{C}$. Since $K \neq \mathbb{R} \cap \overline{\mathrm{Q}}$, the codimension of $K$ in $\mathbb{R} \cap \overline{\mathrm{Q}}$ is $\infty$ (see Artin-Schreier theory [6]). So one can take a subfield $L$ of $\overline{\mathrm{Q}}, L \supset K,[L: K]=l \geq t$. We can enlarge $L$ to $L^{\prime} \subset \overline{\mathrm{Q}}$ such that $i=\sqrt{-1} \in L^{\prime}$ and $L^{\prime} / K$ be a normal extension. So this last $L^{\prime}$ is dense in C. 
We can find $x_{1}, x_{2}, \ldots, x_{t} \in L^{\prime}$ such that $\left|\sigma_{i}\left(x_{i}\right)-\lambda_{i}\right|<\frac{\varepsilon}{3}, i=1,2, \ldots, t$. One can now use the Approximation Theorem (see [1]) to find $x \in L^{\prime}$ such that $\left|x-x_{i}\right|_{\sigma_{i}}=\left|\sigma_{i}(x)-\sigma_{i}\left(x_{i}\right)\right|<\frac{\varepsilon}{3}$ for any $i=1,2, \ldots, t$. This means that $\left|\sigma_{i}(x)-\lambda_{i}\right|<\frac{2 \varepsilon}{3}$ for any $i=1,2, \ldots, t$. But $\left|\bar{e} \sigma_{i}(x)-\bar{\lambda}_{i}\right|<\frac{2 \varepsilon}{3}$ implies that $\left|\sigma_{i}(x)-\lambda_{i}\right|<\frac{2 \varepsilon}{3}$ for all $i=1,2, \ldots, m$. Let us take $\sigma \in G_{K}$ and choose $j \in\{1,2, \ldots, m\}$ such that $\left|f(\sigma)-\lambda_{j}\right|<\frac{\varepsilon}{3}$. Then $\left|f(\sigma)-\sigma_{j}(x)\right|<\varepsilon$ for this $j$. We can enlarge $L^{\prime}$ such that $H=\operatorname{Gal}\left(\overline{\mathrm{Q}} / L^{\prime}\right)$ be good enough for our $\varepsilon>0$. Then $\sigma \in \sigma_{j} H$ means that $\sigma(x)=\sigma_{j}(x)$ because $x \in L^{\prime}$. So $|f(\sigma)-\sigma(x)|<\varepsilon$ or $\left|f(\sigma)-\varphi_{x}(\sigma)\right|<\varepsilon$ for any $\sigma \in G_{K}$. Hence $\left\|f-\varphi_{x}\right\|<\varepsilon$ for an $x \in \overline{\mathrm{Q}}$ and the theorem is completely proved.

REMARK 2. If $K=\mathbb{R} \cap \bar{Q}$, then $G_{K}=\{e, \bar{e}\}$ because $\overline{\mathbb{Q}}=(\mathbb{R} \cap \bar{Q})(i)$. So $\mathcal{C}\left(G_{K}\right)=\mathbb{C} \times \mathbb{C}$ and $\mathcal{C}_{\text {sym }}\left(G_{K}\right)=\mathbb{C}=\{(\alpha, \alpha) \mid \alpha \in \mathbb{C}\} \subset \mathbb{C} \times \mathbb{C}$. But $\widetilde{\mathrm{Q}}_{K}=\mathbb{R}[i]=\mathbb{C}$. Hence Theorem 1 is true even if $K=\mathbb{R} \cap \overline{\mathrm{Q}}$. If $K \subset \mathbb{R} \cap \overline{\mathbb{Q}}$ and $[\mathbb{R} \cap \overline{\mathrm{Q}}: K]<\infty$, then $[\overline{\mathrm{Q}}: K]<\infty$ and $K$ would be real closed in $\overline{\mathrm{Q}}$. Then $\overline{\mathrm{Q}}=K(i)=(\mathbb{R} \cap \overline{\mathrm{Q}})(i)$ and $[\overline{\mathrm{Q}}: K]=[\overline{\mathrm{Q}}: \mathbb{R} \cap \overline{\mathrm{Q}}]=2$ which implies $K=\mathbb{R} \cap \overline{\mathrm{Q}}$ (see Artin-Schreier theory [6]).

THEOREM 2. Let $K \nsubseteq \mathbb{R} \cap \overline{\mathrm{Q}}$ be a subfield of $\overline{\mathrm{Q}}$. Then $\widetilde{\mathrm{Q}}_{K}$ is isometric and isomorphic as a C-algebra with $\mathcal{C}\left(G_{K}\right)$.

Proof. In fact $K \nsubseteq \mathbb{R} \cap \overline{\mathrm{Q}}$ means that $\bar{e} \notin G_{K}$. So the ideas of [13] cannot be applied in this case. But in this case $K$ is dense in $\bar{Q}$ and in C. Since any continuous function $f: G_{K} \longrightarrow \mathrm{C}$ can be uniformly approximated by locally constant functions with a finite number of values, one has only to prove that such a map $g: G_{K} \longrightarrow \mathbb{C}, g(\sigma)=\lambda_{i}$ for any $\sigma \in \sigma_{i} H, i \in\{1,2, \ldots, m\}$, where $G_{K}=\sigma_{1} H \cup \sigma_{2} H \cup \ldots \cup \sigma_{m} H, \sigma_{1}=e,\left[G_{K}: H\right]=m$, is a partition of $G_{K}$ relative to a closed and open subgroup $H$ of $G_{K}$, can be approximated by elements $\varphi_{x}$, with $x \in \overline{\mathrm{Q}}$. Let $\varepsilon>0$ be a small real positive number such that

$$
D\left(\lambda_{i}, \varepsilon\right)=\left\{y \in \mathbb{C}:\left|y-\lambda_{i}\right|<\varepsilon\right\}, i=1,2, \ldots, m
$$

be a disjoint set of balls centered in $\lambda_{i}$. Since $K$ is dense in $\mathbb{C}$, we can take $x_{1}, x_{2}, \ldots, x_{m} \in K$ and $x_{i} \in D\left(\lambda_{i}, \frac{\varepsilon}{2}\right)$ for all $i=1,2, . ., m$. Let $L=\{y \in \overline{\mathrm{Q}}: \mu(y)=y$ for any $\mu \in H\}$ be the fixed field of $H$. Since $\bar{e} \notin G_{K}, \quad\left\{\sigma_{1}, \sigma_{2}, \ldots, \sigma_{m}\right\}$ give rise to independent absolute values $|\cdot|_{\sigma_{1}},|\cdot|_{\sigma_{2}}, \ldots,|\cdot|_{\sigma_{m}}$ on $L$. We use now the Approximation Theorem [1] and 
find $x \in L$ such that

$$
\left|x-x_{i}\right|_{\sigma_{i}}=\left|\sigma_{i}(x)-\sigma_{i}\left(x_{i}\right)\right|<\frac{\varepsilon}{2} \text { for any } i=1,2, \ldots, m .
$$

But $\sigma_{i}\left(x_{i}\right)=x_{i}$ because $x_{i} \in K$, so we get

$$
\left|\sigma_{i}(x)-x_{i}\right|<\frac{\varepsilon}{2}, i=1,2, \ldots, m .
$$

This last inequality together with $\left|x_{i}-\lambda_{i}\right|<\frac{\varepsilon}{2}$ imply

$$
\left|\sigma_{i}(x)-\lambda_{i}\right|<\varepsilon \text { for any } i=1,2, \ldots, m \text {. }
$$

Let us take $\mu \in G_{K}$ and $j \in\{1,2, \ldots, m\}$ such that $\mu \in \sigma_{j} H$, then $\mu(x)=\sigma_{j}(x)$ and $g(\mu)=\lambda_{j}$. Hence $\left|\varphi_{x}(\mu)-g(\mu)\right|<\varepsilon$ for any $\mu \in G_{K}$, so $\left\|\varphi_{x}-g\right\|<\varepsilon$ for an $x \in \overline{\mathrm{Q}}$ and the proof of the theorem is complete.

THEOREM 3. Let $K \subset \mathbb{R} \cap \overline{\mathrm{Q}}$ be a subfield of $\overline{\mathrm{Q}}\left(\bar{e} \in G_{K}\right)$. Then $\mathcal{C}\left(G_{K}\right) \cong \widetilde{\mathbb{Q}}_{K}[\widetilde{i}]$, where $\widetilde{i}$ is the constant function $\widetilde{i}(\sigma)=i=\sqrt{-1}$ for any $\sigma \in G_{K}$.

This is an isomorphism of $\mathbb{R}$ - algebras. If on $\widetilde{\mathrm{Q}}_{K}[\widetilde{i}]$ we consider the componentwise topology induced by $\widetilde{\mathrm{Q}}_{K}$, this last isomorphism is also a homeomorphism.

Proof. The idea of the proof is the same like in [13]. For any $f \in G_{K}$ we consider:

$$
\begin{aligned}
f_{1}(\sigma) & =\frac{\operatorname{Re} f(\sigma)+\operatorname{Re} f(\bar{e} \sigma)}{2}-\frac{\operatorname{Im} f(\sigma)-\operatorname{Im} f(\bar{e} \sigma)}{2 i}, \text { and } \\
f_{2}(\sigma) & =\frac{\operatorname{Re} f(\sigma)-\operatorname{Re} f(\bar{e} \sigma)}{2 i}+\frac{\operatorname{Im} f(\sigma)+\operatorname{Im} f(\bar{e} \sigma)}{2},
\end{aligned}
$$

where $R e(a+i b)=a$ and $\operatorname{Im}(a+i b)=b$. It is not difficult to see that $f_{1}, f_{2} \in \mathcal{C}_{\text {sym }}\left(G_{K}\right)$ and $f=f_{1}+\widetilde{i} f_{2}$.

The following three theorems are the natural generalizations of Theorems 1, 2 and 3. We do not give the proofs of these theorems here because the ideas of the proofs are the same like those used to prove the above last three theorems.

TheOREM 4. Assume $\bar{e} \in G_{K}$ and let $L \subset \bar{Q}$, such that $L / K$ be a subextension of $\overline{\mathrm{Q}} / K$. Let $\widetilde{L}_{K}$ be the completion of $L$ relative to the $K$-spectral norm. Let $H=\left\{\sigma \in G_{K}: \sigma(x)=x\right.$ for all $\left.x \in L\right\}$ be the subgroup of $G_{K}$ which fixes $L$. Then the natural embedding $\varphi_{H}: L \longrightarrow \mathcal{C}\left(G_{K} / H\right)$, where 
$G_{K} / H$ is the quotient topological space of the left classes of $H$ in $G$, $\varphi_{H}(x)=\varphi_{x}$, where $\varphi_{x}(\sigma H)=\sigma(x)$ can be extended to $a \widetilde{K}$-isomorphism and isometry $\bar{\varphi}_{H}: \widetilde{L}_{K} \longrightarrow \mathcal{C}_{\text {sym }}\left(G_{K} / H\right)$.

THEOREM 5. If $\bar{e} \notin G_{K}$, then the same isomorphism and isometry like that in Theorem 2, give rise to a $\widetilde{K}$-Banach algebra isomorphism (isometry) $\widetilde{L}_{K} \cong \mathcal{C}\left(G_{K} / H\right)$.

THEOREM 6. If $\bar{e} \in G_{K}$, then the same isomorphism and isometry like that in Theorem 2, give rise to a $\widetilde{K}$-Banach algebra isomorphism (isometry) $\mathcal{C}\left(G_{K} / H\right) \cong \widetilde{L}_{K}[\widetilde{i}]$.

Theorem 2.2 of [13] can be rewrite in this new general context. We do not prove it because the ideas of the proof are the same like in [13].

Theorem 7. Forany $L \subset \overline{\mathrm{Q}}, K \subset L \subset \overline{\mathrm{Q}}, \widetilde{L}_{K}$ has a topological generic element $x$, i.e. $\widetilde{L}_{K}=\widehat{K[x]}$. In particular, there exist $z \in \widetilde{\mathbb{Q}}_{K}$ such that $\widetilde{\mathrm{Q}}_{K}=\widetilde{K[z]}$.

This last result can be considered as a generalization of the classical primitive element theorem from field theory. It will be useful for future research on the structure of the group $A u t_{\text {cont }}\left(\widetilde{\mathrm{Q}}_{K}\right)$.

3. Let $K$ be a subfield of $\overline{\mathrm{Q}}$ and let $\mathcal{C}\left(G_{K}\right)$ be the C-Banach algebra of all continuous functions defined on $G_{K}=\operatorname{Gal}(\overline{\mathrm{Q}} / K)$, with values in C. For any $f \in \mathcal{C}\left(G_{K}\right)$ one denotes by $\omega(f)=\inf \left\{|f(\sigma)-f(\mu)|: f(\sigma) \neq f(\mu), \sigma, \mu \in G_{K}\right\}$ or $\omega(f)=0$ if $f$ is a constant function. It is easy to see that $\omega(f)>0$ if and only if $f$ has a finite number of distinct values and at least two $(f$ is not constant). Here is a Krasner type lemma [1] for $\mathcal{C}\left(G_{K}\right)$.

LeMma 2. Let $f, g \in \mathcal{C}\left(G_{K}\right)$ such that $f$ is a locally constant map and $\omega(f)>0$. Let us assume that $\|f-g\|<\frac{\omega(f)}{2}$ and that $g(\sigma)=g(\mu)$ for $\sigma, \mu \in G_{K}$. Then $f(\sigma)=f(\mu)$.

Proof. Let us suppose that $f(\sigma) \neq f(\mu)$. Then $\omega(f) \leq|f(\sigma)-f(\mu)| \leq$ $\leq|f(\sigma)-g(\sigma)|+|g(\mu)-f(\mu)| \leq 2\|f-g\|<\omega(f)$, a contradiction. So $f(\sigma)=$ $=f(\mu)$.

Using this result one can obtain an Archimedean version of Krasner Lemma [1] for $\overline{\mathrm{Q}}$. 
Corollary 2. Let $\alpha, \beta \in \overline{\mathrm{Q}}, \alpha \notin K$ such that $\|\alpha-\beta\|_{K}<\frac{\omega(\alpha)}{2}$, where $\omega(\alpha)=\inf \left\{\left|\alpha_{j}-\alpha_{i}\right|: \alpha_{j} \neq \alpha_{i}\right\}$ and $\alpha_{i}, \alpha_{j}$ are all the conjugates of $\alpha=\alpha_{1}$ in $\overline{\mathrm{Q}}$ over $K$. Then $K(\alpha) \subset K(\beta)$.

Proof. Let $\varphi_{\alpha}$ and $\varphi_{\beta}$ be the corresponding locally constant maps in $\mathcal{C}\left(G_{K}\right)$ of $\alpha$ and $\beta$ respectively $\left(\varphi_{\alpha}(\sigma)=\sigma(\alpha), \sigma \in G_{K}\right.$, etc. $)$. Then $\omega\left(\varphi_{\alpha}\right)=\inf \left\{|\sigma(\alpha)-\mu(\alpha)|: \sigma(\alpha) \neq \mu(\alpha), \sigma, \mu \in G_{K}\right\}=\omega(\alpha)$. Since $\left\|\varphi_{\alpha}-\varphi_{\beta}\right\|=$ $=\|\alpha-\beta\|_{K}<\frac{\omega\left(\varphi_{\alpha}\right)}{2}$ we can apply Lemma 2 and obtain that $\varphi_{\beta}(\sigma)=\varphi_{\beta}(\mu)$ $\Longrightarrow \varphi_{\alpha}(\sigma)=\varphi_{\alpha}(\mu)$. Let us take $\sigma=e$, the identity of $G_{K}$. This last implication becomes $\mu(\beta)=\beta \Rightarrow \mu(\alpha)=\alpha$ or $\mu \in \operatorname{Gal}(\overline{\mathrm{Q}} / K(\beta)) \Rightarrow \mu \in \operatorname{Gal}(\overline{\mathrm{Q}} / K(\alpha))$, i.e. $K(\alpha) \subset K(\beta)$.

This last result can be generalized as follows.

THEOREM 8. Let $f, g$ be two locally constant maps in $\mathcal{C}\left(G_{K}\right)$ with $\omega(f)>0$ ( $f$ not constant). Let $\Omega \subset \mathbb{C}$ be a subfield of $\mathbb{C}$ such that $f(\sigma)$ and $g(\sigma) \in \Omega$ for all $\sigma \in G_{K}$. We assume that $\|f-g\|<\frac{\omega(f)}{2}$. Then $\Omega[f]$ $\subset \Omega[g]$ in $\mathcal{C}\left(G_{K}\right)$. Here $\Omega[f]=\left\{a_{0}+a_{1} f+\ldots+a_{t} f^{t} \mid a_{0}, a_{1}, \ldots, a_{t} \in \Omega\right\}$ and $f^{i}(\sigma)=[f(\sigma)]^{i}$ for any $\sigma \in G_{K}$ and $i \in \mathbb{N}$. Moreover, if $\|f-g\|<\frac{1}{2} \min \{\omega(f), \omega(g)\}$ and if $\omega(g)>0$, then $\Omega[f]=\Omega[g]$.

Proof. Since $G_{K}$ is a compact group $f$ and $g$ have a finite number of values. Let $m$ be the number of distinct values of $g: g\left(\sigma_{1}\right)=\lambda_{1}$, $g\left(\sigma_{2}\right)=\lambda_{2}, \ldots, g\left(\sigma_{m}\right)=\lambda_{m} \in \Omega$. Then we can solve the linear system of $m$ equations and $m$ unknowns $A_{0}, A_{1}, \ldots, A_{m-1}$ :

$$
\begin{aligned}
& f\left(\sigma_{1}\right)=A_{0}+A_{1} \lambda_{1}+A_{2} \lambda_{1}^{2}+\ldots+A_{m-1} \lambda_{1}^{m-1} \\
& f\left(\sigma_{2}\right)=A_{0}+A_{1} \lambda_{2}+A_{2} \lambda_{2}{ }^{2}+\ldots+A_{m-1} \lambda_{2}{ }^{m-1} \\
& f\left(\sigma_{m}\right)=A_{0}+A_{1} \lambda_{m}+A_{2} \lambda_{m}{ }^{2}+\ldots+A_{m-1} \lambda_{m}{ }^{m-1}
\end{aligned}
$$

The determinant $\Delta$ of the system is equal to $\prod_{i<j}\left(\lambda_{i}-\lambda_{j}\right) \neq 0$. So $A_{i}=\frac{\Delta_{i}}{\Delta} \in \Omega$ for all $i=0,1, \ldots, m-1$. Now, if $g(\sigma)=g\left(\sigma_{i}\right)$ for an $i=1,2, \ldots, m$, then $f(\sigma)=f\left(\sigma_{i}\right)$ (see Lemma 2 ) for the same $i$. Hence, for 
these $\left\{A_{i}\right\}, i=1,2, \ldots, m$ we can write:

$$
\begin{gathered}
f(\sigma)=A_{0}+A_{1} g(\sigma)+A_{2} g^{2}(\sigma)+\ldots+A_{m-1} g^{m-1}(\sigma), \text { i.e. } \\
f=A_{0}+A_{1} g+A_{2} g^{2}+\ldots+A_{m-1} g^{m-1}, \text { or }
\end{gathered}
$$

$f \in \Omega[g]$. Therefore $\Omega[f] \subset \Omega[g]$. The last statement is simply obtained be changing $f$ and $g$, one to each other.

Corollary 3. Let $B$ be a C-subalgebra of $\mathcal{C}\left(G_{K}\right)$ and let $f$ be a nonconstant locally map in $\widetilde{B}$, the topological closure of $B$ in $\mathcal{C}\left(G_{K}\right)$. Then $f \in B$.

Proof. Since $B$ is dense in $\widetilde{B}$, let $g$ be in $B$ such that $\|f-g\|<\frac{\omega(f)}{2}$. By Theorem 8 we obtain that $\mathrm{C}[f] \subset \mathrm{C}[g] \subset B$, i.e. $f \in B$.

Proposition 2. Let $K \subset L \subset \overline{\mathrm{Q}}$ be subfields of the algebraic closure of Q. Let $\widetilde{\mathrm{Q}}_{K}$ be the $K$-spectral closure of $\overline{\mathrm{Q}}$ and let $\widetilde{L}_{K} \subset \widetilde{\mathrm{Q}}_{K}$ the topological closure of $L$ in $\widetilde{\mathrm{Q}}_{K}$ (relative to the $K$-spectral norm). Then $\widetilde{L}_{K} \cap \overline{\mathrm{Q}}=L$.

Proof. Let $x \in \widetilde{L}_{K} \cap \overline{\mathrm{Q}}$. If $x \in K$, then $x \in L$. If $x \notin K$, then $\omega(x)=\min \left\{\left|x_{j}-x_{i}\right|: x_{j} \neq x_{i}\right\}$ where $x_{i}$ are all the $K$-conjugates of $x=x_{1}$ in $\overline{\mathrm{Q}}$, is not zero. Let $y \in L$ such that $\|x-y\|_{K}<\frac{\omega(x)}{2}$. Then $K(x)$ $\subset K(y) \subset L$ (see Corollary 2). Hence $x \in L$ and so $\widetilde{L}_{K} \cap \overline{\mathrm{Q}}=L$.

We recall that the mapping $\varphi: \overline{\mathrm{Q}} \rightarrow \mathcal{C}\left(G_{K}\right)$, defined by $\varphi(x)=\varphi_{x}$, where $\varphi_{x}(\sigma)=\sigma(x)$ for any $x \in \overline{\mathrm{Q}}$ and $\sigma \in G$, is an isometric $K$-embedding of $\overline{\mathrm{Q}}$ into $\mathcal{C}\left(G_{K}\right)$.

Proposition 3. Let $A$ be a closed $K$-subalgebra of $\mathcal{C}\left(G_{K}\right)$. Then $A \cap \varphi(\overline{\mathrm{Q}})$ is a subfield of $\varphi(\overline{\mathrm{Q}})$.

Proof. Let $B=A \cap \varphi(\bar{Q})$ and let $x \in B \backslash\{1\}$. Since $\mathrm{Q} \subset B$ we may suppose that $\|x\|_{K}<1$. Since $\mathcal{C}\left(G_{K}\right)$ is a Banach algebra over $\mathrm{C}$ (see [5] or [18]) and because $A$ is closed, the series $1+x+x^{2}+\ldots=y \in A$. But $y=\frac{1}{1-x}$, so $1-x$ is invertible in $B$ (because in $\varphi(\bar{Q})$ it is !). So we just proved that for any $x \in B, x \neq 1,1-x$ has an inverse in $B$. Let $\mathbf{m}$ be a maximal ideal of $B$ and let $z \neq 0, z \in \mathbf{m}$. Then $z=1-(1-z)$ has an inverse in $B$, i.e. $\mathbf{m}=B$, thus $B$ is a subfield of $\varphi(\bar{Q})$. in $\widetilde{\mathrm{Q}}_{K}$.

Propositions 2 and 3 can be viewed as the first steps to a Galois theory 
Acknowledgments. We express our gratitude to the referee for his patience in reading our paper and for his (or her) opinions which directly led us to improve the first version of the article (in particular Theorem 8).

\section{REFERENCES}

[1] E. Artin, Algebraic numbers and functions, Gordon and Breach, Science Publications, New York, Paris, London, 1967.

[2] V. Alexandru - N. Popescu - A. Zaharescu, On the closed subfields of $\mathrm{C}_{p}$, J. Number Theory, 68, no. 2 (1998), pp. 131-150.

[3] V. Alexandru - N. Popescu - A. Zaharescu, Trace on $\mathbb{C}_{p}$, J. Number Theory, 88 (2001), pp. 13-48.

[4] A. D. R. Choudary - A. Popescu - N. Popescu, On the structure of compact subsets of $\mathrm{C}_{p}$, Acta Arith., 123, no. 3 (2006), 253-266.

[5] L. Gilman - M. Jerison, Rings of continuous functions, D. van Nostrand Company, Inc., 1960.

[6] N. Jacobson, Lectures in Abstract Algebra III. Theory of Fields and Galois Theory, D. van Nostrand Company, Inc., Princeton, New York, 1964.

[7] J. NeuKIRCH, Algebraic number theory, Grund. Math. Wiss., Vol. 322 (Springer Verlag, 1999).

[8] A. POPESCU, On some topological representations of the absolute Galois group, JP of Algebra, N. Th. \& Appl., 4 (1) (2001), pp. 103-112.

[9] E. L. Popescu, Spectral extensions, Anal. Univ. Bucuresti Mat., 51 (2002), pp. 159-168.

[10] N. Popescu, Galois action on plane compacts, in: Proc. 35th Sympos. on Ring Theory and Representation Theory (Okayama, 2002), Okayama,2003, pp.113-120.

[11] V. PAsol - A. Popescu - N. Popescu, Spectral norms on valued fields, Math. Z., 238 (2001), pp. 101-114.

[12] L. Popescu - N. Popescu - C. VRACIU, Completion of the spectral extension of a p-adic valuation, Rev. Roumaine Math. Pures Appl., 46 (2001), pp. 805-817.

[13] A. Popescu - N. Popescu - A. Zaharescu, On the spectral norms of algebraic numbers, Math. Nach., 260 (2003), pp. 78-83.

[14] A. Popescu - N. Popescu - A. Zaharescu, Galois structures on plane compacts, J. of Algebra, 270 (1) (2003), pp. 238-248.

[15] A. Popescu - N. Popescu - A. Zaharescu, Trace series on $\widetilde{Q}_{K}$, Results Math., 43 (2003), pp. 331-342.

[16] A. Popescu - N. Popescu - A. Zaharescu, Transcendental divisors and their critical functions, Manuscripta Math., 110 (2003), pp. 527-541.

[17] A. Popescu - N. Popescu - A. Zaharescu, A Galois theory for the Banach algebra of continuous symmetric functions on absolute Galois groups, Results Math., 45 (2004), pp. 349-358.

[18] W. Rudin, Real and Complex Analysis, McGraw-Hill International editions Mathematics Series (New York, Dusseldorf), 1987.

[19] A. Zaharescu, Some notes on $\widetilde{\mathrm{Q}}$, a letter to N. Popescu and A. Popescu, January 6, 2000.

Manoscritto pervenuto in redazione l'11 novembre 2007. 
